\title{
Topography evaluation methods for concrete substrates: parametric study
}

\author{
L. Courard \\ University of Liège, Belgium
}

\author{
A. Garbacz \\ Warsaw University of Technology, Poland \\ B. Bissonnette \\ Laval University, Québec, Canada
}

\begin{abstract}
Research projects performed at the University of Liege, Laval University and the Warsaw University of Technology have pointed out the importance of taking care about the surface roughness of concrete substrate with regards to the adhesion of repair materials. This paper wants to make a State-of-Art on this specific problem: surfometry methods, interface analysis, adhesion measurements, ...etc are presented, interpreted and compared. Surfology, which is a more wide concept, contributes to understand what will make the contact effective or not, and allow interactions of variable intensities between the materials. Different scales of observation - micro to macro - are needed to exactly represent what happens when materials are put into contact.
\end{abstract}

\section{INTRODUCTION}

In addition to adhesion and cohesion, another parameter often considered affecting the tensile bond between repair material and existing concrete is the substrate roughness. Nevertheless, this subject has been controversial for years. In fact, some reported bond test results have shown that surface roughness has only a minor influence on the tensile bond. For instance (Silfwerbrand, 1990), bond to rough water jetted surface was compared with bond to smooth sandblasted surface. It was concluded that there could be a roughness "threshold value" beyond which further improvement on the roughness would not enhance bond strength (Courard et al., 2010). According to these test results, the "threshold value" ought to be close to the surface roughness of the typical sandblasted surfaces (Courard et al., 2009). However, it remains the opinion of many specialists in the industry that a rougher surface is beneficial to bond strength. Since roughness directly depends on the surface preparation method, the proposed research is intended to shed new light on the subject and ultimately resolve the controversy.

According to American National Standards Institute, the methods for measuring roughness and surface texture can be classified into three types: contacting methods, taper sectioning, and optical (noncontacting) methods.

Taper sectioning is used in metallurgy and involves cutting across a surface at a low angle $\alpha$ to physically amplify the height of asperities by $\operatorname{ctg} \alpha$ (Sherrington et al., 1988).
Among the contacting methods there are stylus type profilometers, tactile tests, measurement of kinetic friction, measurement of static friction, use of rolling ball measurements, and measurement of the compliance of a metal sphere with a rough surface.

Optical (non-contacting) methods include optical reflecting instruments, light microscopy, electron microscopy, speckle metrology, interferometry and laser profilometry (Fukuzawa et al., 2001).

A variety of approaches have been set all over the years to characterize the surface roughness of concrete: evaluation of the proportion of the surface occupied by aggregates, measurement of the maximum roughness amplitude (Courard, 1998), adhesion tests (Pretorius et al., 2001 and Garbacz et al., 2005), calculation of surface parameters based on image analysis or on microscopic observations, etc. However, these methods are unable to provide a sufficiently detailed representation of the actual surface profile for the calculation of morphological and statistical parameters, and are not user-friendly under field conditions. In order to achieve a reliable quantitative analysis of superficial concrete morphology after surface preparation (Bissonnette et al., 2006), different profilometry and surfometry techniques can be used (Perez et al., 2005). The data obtained with such techniques enable a real quantitative assessment of the surface profile by means of statistical parameters calculated from the total superficial profile and from the filtered waviness (low frequency/macro roughness) and roughness (high frequency/microroughness) profiles (Courard et al., 2003 and Courard et al., 2004). Some of these parameters 
- for instance the profile arithmetic mean and the flatness coefficient - are particularly discriminating both for the shape of valleys and peaks, as well as for their amplitude and frequency.

The selected characterization techniques were compared for effectiveness, accuracy, consistency and field applicability. The following techniques were analyzed on a comparative basis:

- the evaluation of surfaces with sand patch test according to ASTM E965 (very close to EN 13036-1: 2002);

- mechanical profilometry technique, in which a high-precision extensometer is moved all over the surface to obtain a 3-D mapping ( $\mathrm{x}$, $\mathrm{y}, \mathrm{z}$ coordinates), from which morphological parameters are computed;

- laser technique, where the superficial elevation (distance from the laser beam source) of each point is calculated on the basis of the laser beam transit time;

- opto-morphometry technique, based on the observation and analysis of the shadow produced by the superficial roughness of the surface (Moiré fringe pattern principle);

- microscopic methods;

- concrete surface profiles (CSP) plaques 03732).

The aim of this paper is the identification of the different available techniques (laboratory and field use), as well as their comparison for evaluating relevant quantitative roughness characteristics.

\section{METHODS FOR SURFACE ROUGHNESS EVALUATION}

\subsection{Visual observation}

The visual evaluation of surface roughness is the most simple but rather subjective way of evaluation. The systematic approach for visual surface qualification was proposed by ICRI (ACI).

Table 1. Concrete surface treatment methods and corresponding Concrete Surface Profile (CSP)

\begin{tabular}{lc}
\hline Surface preparation methods & CSP \\
\hline Detergent scrubbing & 1 \\
Low-pressure water cleaning & 1 \\
Acid etching & $1-3$ \\
Grinding & $1-3$ \\
Abrasive (Sand) blasting & $2-5$ \\
Steel shotblasting & $3-8$ \\
Scarifying & $4-9$ \\
Needle scaling & $5-8$ \\
Hydrodemolition & $6-9$ \\
Scabbling & $7-9$ \\
Flame blasting & $8-9$ \\
Milling/rotmilling & 9 \\
\hline
\end{tabular}

The reference plaques called Concrete Surface Profile - CSP (Tab.1) represented concrete surface after typical surface treatment commonly used in the field. The details are given in ICRI Guidelines no. 03732 .

\subsection{Sand patch test}

The sand patch tests described in ASTM E965 (very close to EN 13036-1:2002) is one of the most commonly methods used for examination of macrotexture depth of concrete surfaces, mainly road and airfield pavements. This direct volumetric method consists in careful application of a given volume of granular materials (a glass spheres or sand) of given granulometry onto surface and subsequent measurement of the total area covered. Poured quantity of granular materials is spreading in a circular pattern with the rubber disc spreading tool, kept flat on the surface, into a circular patch with the surface depressions filled to the level of the peaks. As the sand is spread, it fills the low spots in the pavement surface. When the sand cannot be spread any further, the diameter of the resulting circle is measured (Fig.1). The surface roughness is characterized by so called the Mean Texture Depth (MTD) calculated from the following formula:

$\mathrm{MTD}=4 \mathrm{~V} / \pi \mathrm{D}^{2}[\mathrm{~mm}]$

where:

$\mathrm{V}=$ volume of a granular material $\left[\mathrm{mm}^{3}\right]$

$\mathrm{D}=$ diameter of circle covered by a granular material [mm]
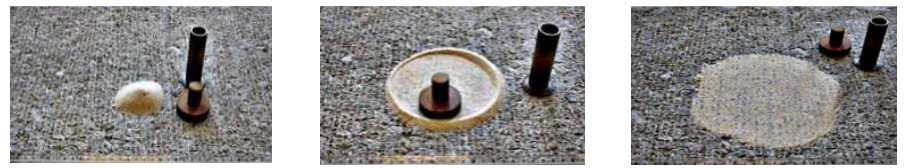

Figure 1. Sand patch test for surface macrotexture depth evaluation

According to ASTM E965 and EN 13036-1 standard a glass spheres of $250 / 180 \mu \mathrm{m}$ are recommended as the granular materials. However, silica sand is also used.

Similar way of surface roughness evaluation is proposed in the European Standard EN 1766:2000 in the case concrete substrate preparation prior repair. Silica sand with granulometry $100 / 50 \mu \mathrm{m}$ is recommended for evaluation. Surface Roughness Index is calculated from the formula:

$\mathrm{SRI}=\left(\mathrm{V} / \mathrm{d}^{2}\right) .1272$

Where symbols like in eq. 1 and $V=25 \mathrm{ml}$ is recommended

The advantages of sand patch method are speed, non-destructive character, applicability in situ but 
the surface has to be protected from wind and rain. The biggest limitations are board of validity (from 0.25 to $5 \mathrm{~mm}$ ) and possibility of application only at horizontal surfaces.

\subsection{Profilometry methods}

\subsubsection{Mechanical profilometry}

The deviations of surface geometry are detected by a sensor (stylus) that moves along the surface. The gauge turns the vertical deflection of the stylus as a function of position into the electrical signal which is registered by the computer and in the end a surface profile is obtained (Fig.2a). There is a possibility of regulation of the distance between measurement points to get better precision. Geometry (round or conical) and size (radius) of the stylus extremity are of prime significance for real profile restitution because, some profile of small wavelengths will not be registered, if the diamond cone radius is too large (Fig.2b).

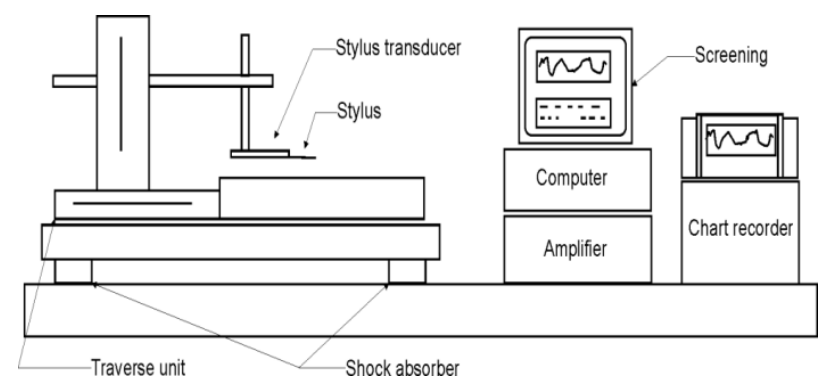

a)

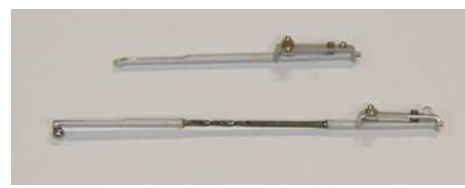

b)

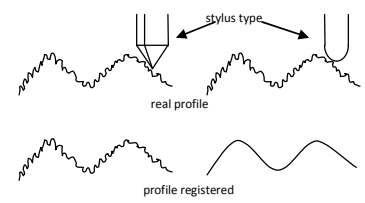

c)
Figure 2. Mechanical profilometer: a) a scheme of device, b) stylus adopted for concrete surface roughness evaluation, c) effect of stylus type on registered profile (Courard et al., 2004).

\subsubsection{Laser profilometry}

Laser profilometry method is based on a laser distance measurement by optical displacement meter. In this technique a principle referred to as optical triangulation. Optical triangulation uses a light source (commonly a diode laser), imaging optics, and a photodetector. As shown in Figure 1, a diode laser is used to generate a collimated beam of light, which is then projected onto a target surface. A lens focuses the spot of reflected laser light onto a photodetector, which generates a signal that is proportional to the spot's position on the detector. As the target surface height changes, the image spot shifts due to the parallax. To generate a three dimensional image of the part's surface, the sensor scans in two dimensions, generating a helical set of radius data that represent the inside surface topography of the tube. Software then generates a color image of the inside surface of the tube. The idea is taken from an old comb method and it is very simple but instead of the needles there is an emitter of a laser signal moving along the surface and measuring distance to the surface (Fig.3).

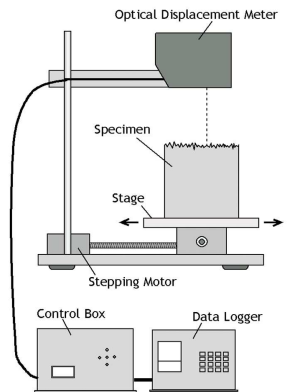

a) laser profilometry equip-

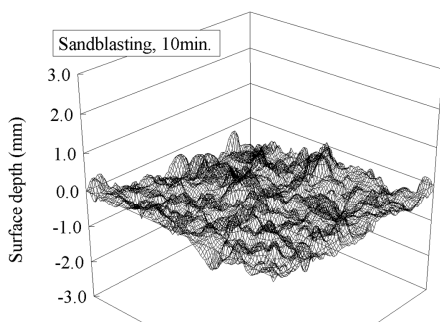

b) result screen of laser profilometry
Figure 3. Laser profilometry with optical displacement meter (Fukuzawa et al., 2001)

\subsubsection{Opto-morphology profilometry}

Above presented two profilometry methods are used only in laboratory conditions (Courard et al., 2007). Recently, works are focused on development optic based methods which can be used insite. The "moiré" projection technique which belongs to an interferometrical measurement method can be considered as the technique useful for this purpose. The "moiré» phenomenon appears when two networks of light rays, made of equidistant lines - alternatively opaque and transparent -, are superimposed. The technique of identification of relief is based on the deformation's measurement of a parallel fringes pattern projected on a surface (Fig. 4). The moiré's fringes are similar to level lines representing the variations height of the object. By projecting a network of parallel fringes on a plane surface, this network will not be deformed. When projected on an unspecified form, this same network will be deformed according to the level of rise in this form. The main principle of the test consists in the comparison of two images having two different moiré networks. The first image is the image reference: it is an image of the network of not deformed parallel fringes. The second image contains the projected network deformed according to the analyzed form. An algorithm analyzes the image and compares the grid of calibration and the deformed grid.

\subsubsection{Characterization of surface roughness}

The result of roughness measurement is usually the image of the profile. To analyze the influence of the treatment on the surface it is necessary to quantify mathematically and statistically the shape of the surface by means of several parameters. 

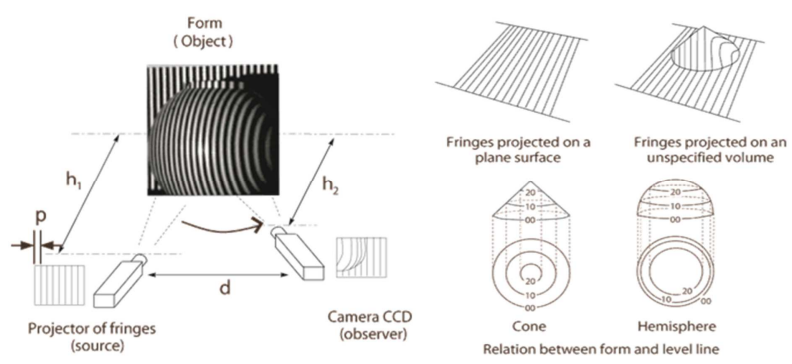

Figure 4. Moire projection principle (Perez et al., 2009)

Another approach is surfometry - a surface metrology of profile extended to $3 \mathrm{D}$. The profilometer is used to get many parallel profiles at close spacing between them. The result is analyzed not in one direction but two perpendiculars $(\mathrm{x}, \mathrm{y})$ and get a $3 \mathrm{D}$ visualization of the surface. This method gives the quantification of surface geometry independent of the anisotropy.

\section{Signal filtration}

The total profile presents a combination of two composites: waviness and roughness. In surface characterization methods they have to be separate. In mechanical profilometry a filtration by differentiating the diameters of the stylus is often used. It is also possible to filtrate the result in mathematic way (Perez et al., 2005).

\section{COMPARISON OF TECHNIQUES}

\subsection{Mechanical vs. optical profilometry}

Concrete substrates $(300 \times 300 \times 50 \mathrm{~mm})$ of $\mathrm{C} 20 / 25$ class were made from the concrete mix: CEM I 32.5, 2/8 limestone, $0 / 2$ quartz sand. The following types of mechanical treatments were used to prepare the concrete substrates:

- $\quad$ grinding (GR),

- sandblasting (SB),

- shotblasting (SHB35 and SHB45, with treatment time of 35 , and $45 \mathrm{~s}$, respectively),

- hand (HMIL) and mechanical (MMIL) milling.

The surface roughness after various treatments was characterized with the following methods:

- mechanical profilometry: the commercial profilometer developed for metal surface testing has been adopted for evaluation of a concrete substrate by changing the stylus (Courard et al., 2003). In the first step, the roughness of the profile was analyzed. In this case a stylus with a diamond sphere radius of $6 \mu \mathrm{m}$ was used. The length of measurement was $8 \mathrm{~mm}$ and the filter used to separate roughness from the total profile was fixed to $0.8 \mathrm{~mm}$. The measurement of waviness was made with another stylus $79 \mathrm{~mm}$ long and a diamond of $1.5 \mathrm{~mm}$ radius. The length of the measurement was enlarged to $30 \mathrm{~mm}$ or more. The filter to separate waviness from the total profile was classically chosen at 0.8 $\mathrm{mm}$.

- laser profilometry: the concrete surface was tested with a commercial laser profilometer working in the laser beam triangulation mode with a vertical accuracy of $1 \mu \mathrm{m}$ and maximum angle of surface measurement of $90^{\circ}$. A correction of the surface image (local lack of height data) was necessary. The missing data were approximated using a smooth shape calculated from the neighbours.

- Sand patch method (acc. EN 1766) - the surface roughness described using the so called Surface Roughness Index - SRI. This consists in spreading silica sand $(50-100 \mu \mathrm{m})$ onto the surface, making a circle and measuring its average diameter, which defines the SRI value;

- microscopic method - the stereological parameters are: surface roughness ratio, RS, profile roughness ratio, RL and fractal dimension, $\mathrm{Db}$. They were determined with vertical sectioning methods for the profile images registered with a light microscope at magnification 10x. Samples for microscopic observation of $20 \times 50 \mathrm{~mm}$ were cut from concrete plate of 300x300 mm (Fig.1a). The total length of examined profile was $350 \mathrm{~mm}$ for each substrate type.

In the case of laser profilometry (Fig. 3), the area of 10x $30 \mathrm{~mm}$ was scanned along parallel lines with a distance of $50 \mu \mathrm{m}$ between subsequent lines (Garbacz et al., 2006). In the case of mechanical profilomtery the surface was scanned along three lines of $30-40 \mathrm{~mm}$ long. The registered profile was first transformed to remove the effect of the profile orientation ("shape" filtration). The total profile obtained was next filtered and divided into low and high frequencies to separate parameters of waviness and roughness, respectively. The filter used to separate waviness from the total profile was classically chosen at $0.8 \mathrm{~mm}$ for both methods. The total height of the profile, $\mathrm{Xt}$, arithmetic mean of the deviations of the profile from the mean line, $\mathrm{Xa}$, and maximum depth of valleys, $\mathrm{Xv}$, were selected for the surface geometry characterization in the case of all levels of filtration (Perez et al., 2009), i.e. for the total (X=P), waviness $(\mathrm{X}=\mathrm{W})$ and roughness $(\mathrm{X}=\mathrm{R})$ profiles. Additionally, the Abbott's curve parameters were determined. The shape of Abbott's curve is characterized by three parameters (Courard et al., 2003):

- $\mathrm{Cr}$ - relative height of the peaks;

- $\mathrm{Cf}$ - depth of the profile, excluding high peaks and holes;

- $\mathrm{Cl}$ - relative depth of the holes. 
In the further text indexes " $p$ " and "s" denote parameters measured by mechanical and laser profilometer respectively.

Figures 5 and 6 present a comparison between Surface Rough Index and parameters measured on the base of profilometry techniques: an equivalent correlatio exists between the mean waviness obtained by means of the two profilometry techniques and SRI, respectively.

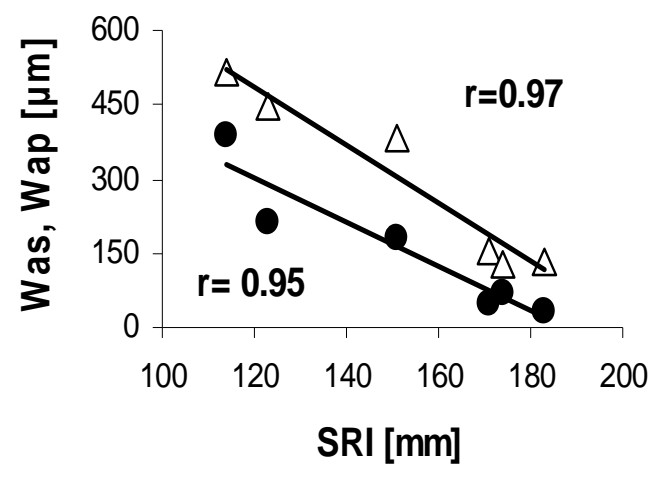

Figure 5. Surface Rough Index vs arithmetic mean of waviness; $(\mathrm{p}, \Delta)$ and $(\mathrm{s}, \bullet)$ for mechanical and laser profilometers (Garbacz et al., 2006)

Similar conclusions may be given for Abbott's parameters (Fig. 6).

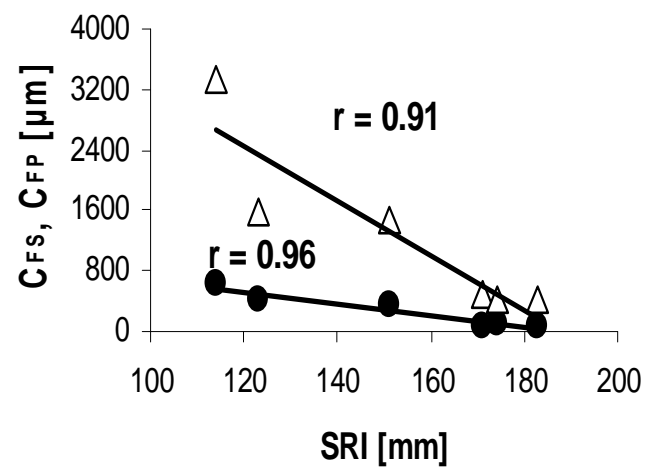

Figure 6. Surface Rough Index vs Abbott's parameters; $(\mathrm{p}, \Delta)$ and $(\mathrm{s}, \bullet)$ for mechanical and laser profilometers (Garbacz et al., 2006)

The mean roughness values are close to each other for the treatment types and the both profilometry methods.

The total height and the mean value of the waviness profile (Fig. 8) measured with the laser profilometry are $1.3-4.3$ times higher than the ones deduced from the mechanical method. In the case of the parameters of Abbott's curve (Fig.9) this ratio was even 7 times higher.

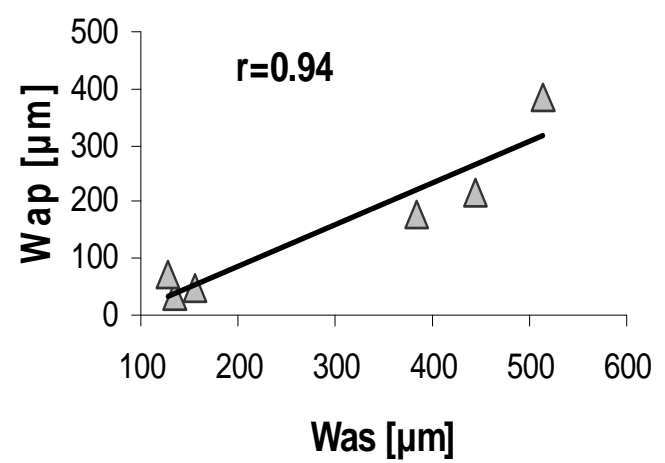

Figure 7. Comparison of mean waviness; "p" and "s" for mechanical and laser profilometers (Garbacz et al., 2006)

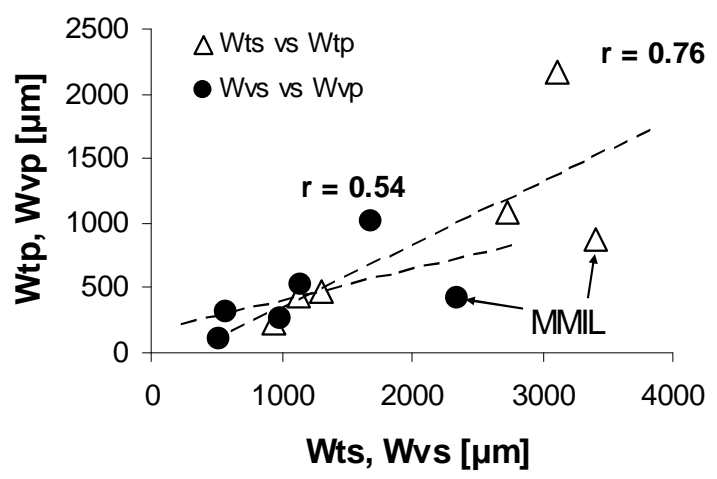

Figure 8. Comparison of maximum peak height and maximum height waviness; "p" and "s" for mechanical and laser profilometers (Garbacz et al., 2006)

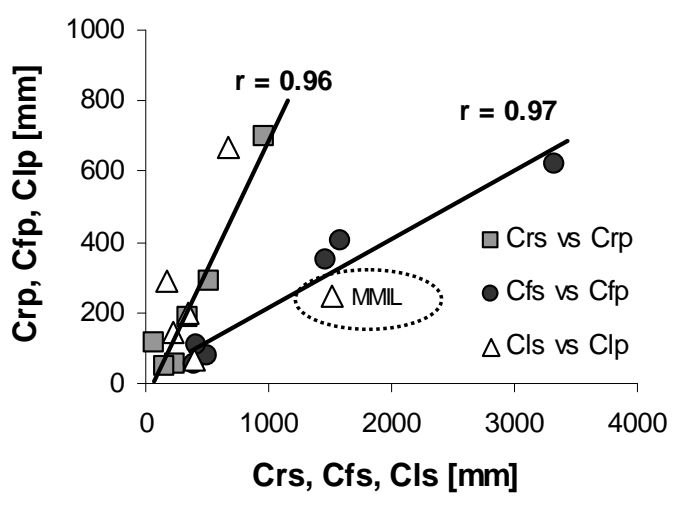

Figure 9. Abbott's parameters; "p" and "s" for mechanical and laser profilometers (Garbacz et al., 2006)

\section{CONCLUSIONS}

The results of surface geometry characterization (Tab.1) with the four methods can be summarized as follow:

- the geometrical parameters determined for both macroscopic level (SRI value) and microscopic level (RS, RL ratios and waviness 
parameters) generally indicate that a higher roughness was obtained after shot blasting for $45 \mathrm{~s}$ and a lower roughness by grinding;

- in the case of the profilometry methods, the waviness parameters are about 5\% (mechanical profilometry) and 9\% (laser profilometry) smaller than the one corresponding to the total profile. This confirms that the global shape of the profile has been preserved through the waviness filtration;

- the mean roughness values are close to each other for the treatment types and the both profilometry methods (Rap $=17 \pm 2$ and Ras $=19 \pm 7$, respectively). However, the total height of the roughness profile determined with laser profilometry was $2.8-5.5$ times exceeding the one obtained with mechanical profilometry with the same filtration method.

- both the total height and the mean value of the waviness profile measured with the laser profilometry are $1.3-4.3$ times higher than the ones deduced from the mechanical method. In the case of the parameters of Abbott's curve this ratio was even 7 times higher. However, values of these ratios do not correspond to the waviness level;

- the values of fractal dimension, $\mathrm{Db}$ determined with the microscopic method are close to those obtained for various types of concrete $(\mathrm{D}=1.03-1.25)$. Range of $\mathrm{Db}$ values is higher in comparison with the surface fractal dimension, DS, obtained with laser profilometry. The low scattering of DS value is caused by measurements for surface area with relatively low irregularity. However, the values obtained of DS are higher than the values that have been determined for fracture surfaces (DS $=2.02-2.3$ ) of various types of concretes and close to those determined for, e.g. steel after surface treatment by grinding.

\section{REFERENCES}

Bissonnette, B., Courard, L., Vaysburd, A. and Bélair, N. 2006. Concrete removal techniques: influence on residual cracking and bond strength. Concrete International, 28(12): 4955.

Courard, L. 1998. Parametric Definition of sandblasted and polished Concrete Surfaces, in: IXth International Congress on Polymers in Concrete, Bologna, Italy (ICPIC, Ed. P. Sandrolini,): 771-778.

Courard, L. and Nélis, M. 2003. Surface analysis of mineral substrates for repair works: roughness evaluation by profilometry and surfometry analysis. Magazine of Concrete Research 55(4): 355-366.

Courard, L., Garbacz, A. and Gorka, M. 2004. Concrete surface treatments quantification by means of mechanical profilometry. in: ICPIC, XIth International Congress on Polymers in Concrete (Ed. M. Maultzsch, Federal Institute for
Materials Research and Testing), Berlin, Germany (2-4 June 2004): 125-132.

Courard, L., Schwall, D. and Piotrowski, T. 2007. Concrete surface roughness characterization by means of optomorphology technique. Monography: Adhesion in Interfaces of Building Materials: a Multi-Scale Approach (AMSR Advances in Material Science and Restoration, Eds. L. Czarnecki and A. Garbacz, Aedificio Publishers): 107-116.

Courard, L., Michel, F., Schwall, D., Van der Wielen, A., Garbacz, A., Piotrowski, T., Perez, F. and Bissonnette, B. Surfology: concrete substrate evaluation prior to repair. Materials Characterization: computational methods and experiments IV (eds. A. Mammoli and C.A. Brebbia, Wessex Institute of Technology Press). The New Forest (U.K.), 17-19 June, 2009: 407-16.

Courard, L., Vaysburd, A. and Bissonnette, B. Repair of concrete industrial floors with bonded cement-based material overlays: some recommendations. Seventh International Colloquium Industrial Floors '10, (Ed. K. Littmann, Technische Akademie Esslingen), Ostfildern/Stuttgart, Germany (2010): 617-622.

Fukuzawa, K., Mitsui, M. and Numao, T. 2001. Surface roughness indexes for evaluation of bond strengths between CRFP sheet and concrete. In: Xth International Congress on Polymers in Concrete (ICPIC 01, Ed. D. Fowler), Honolulu, Hawaï: p12.

Garbacz A., Courard L., Kostana K. 2006. Characterization of concrete surface roughness and its relation to adhesion in repair systems, Materials Characterization, 56: 281-289

Garbacz, A., Courard, L., and Gorka, M. 2005. Effect of concrete surface treatment on adhesion in repair systems. Magazine of Concrete Research 57(1):49-60.

Perez, F., Courard, L., Bissonnette, B., Garbacz, A. and Gorka, M. Two different techniques for the evaluation of concrete surface roughness. In: ICCRRR 2005 International Conference on Concrete Repair, Rehabilitation and Retrofitting (Eds. H. Beushausen, F. Dehn and M.G. Alexander, 2006 Taylor \& Francis Group, London), Cape Town, South Africa (2005): 1015-1020.

Perez, F., Bissonnette, B.and Courard, L. 2009. Combination of mechanical and optical profilometry techniques for concrete surface roughness characterization. Magazine of Concrete Research 61(6): 389-400.

Pretorius, J. and Kruger, D. 2001. The influence of surface roughness on the bond strength of concrete repairs. In: Xth International Congress on Polymers in Concrete (ICPIC 01, Ed. D. Fowler), Honolulu, Hawaï: p13.

Sherrington, I. and Smith, E.H. 1988. Modern measurement techniques in surface metrology - Part I: Stylus instruments, electron microscopy and non-optical comparators. Wear 125: $271-288$.

Sherrington, I. and Smith, E.H. 1988. Modern measurement techniques in surface metrology - Part II: Optical instruments Wear, 125: 289-308.

Silfwerbrand J. 1990. Improving Concrete Bond in repaired Bridge Decks. Concrete International 12 (9): 61-66.

\section{AKNOWLEDGMENTS}

This research project was supported by the scientific cooperation program between foreign agencies of Wallonia-Brussels International (Belgium), Québec (Canada) and Poland. 